\title{
La constitution d'un glossaire dans un cours d'anglais informatique : prétexte ou fin en soi ?
}

Nadine Pasternak-Eboueya

\section{(2) OpenEdition}

Journals

Édition électronique

URL : http://journals.openedition.org/asp/4402

DOI : 10.4000/asp.4402

ISSN : 2108-6354

Éditeur

Groupe d'étude et de recherche en anglais de spécialité

Édition imprimée

Date de publication : 1 mars 1993

Pagination : 519-529

ISSN : 1246-8185

Référence électronique

Nadine Pasternak-Eboueya, «La constitution d'un glossaire dans un cours d'anglais informatique prétexte ou fin en soi ? », ASp [En ligne], 1 | 1993, mis en ligne le 04 juin 2014, consulté le 19 avril 2019. URL : http://journals.openedition.org/asp/4402 ; DOI : 10.4000/asp.4402

Ce document a été généré automatiquement le 19 avril 2019

Tous droits réservés 


\title{
La constitution d'un glossaire dans un cours d'anglais informatique : prétexte ou fin en soi ?
}

\author{
Nadine Pasternak-Eboueya
}

1 Cet article est l'aboutissement d'un travail que j'ai entrepris cette année avec les étudiants de Maîtrise de sciences et techniques option Génie informatique. Le désarroi que j'ai connu lorsque l'on m'a demandé de dispenser un cours d'anglais scientifique dans un domaine de référence pour lequel je n'étais pas formée (Vision, Traitement de l'image, Infographie) est à l'origine de cette expérience que j'ai tentée sur six mois. Il fallait essayer de construire un enseignement rigoureux et approprié. J'acceptais le défi.

\section{Introduction}

2 Les quelques questions que je vais évoquer ici méritaient d'être posées; il s'agissait de définir le public et le profil des étudiants; d'identifier leurs besoins; de cerner le domaine; de répertorier les ressources; de fixer les objectifs et de réfléchir sur les stratégies à mettre en œuvre pour les atteindre.

3 Cette première promotion est constituée d'un groupe de 26 étudiants qui viennent d'horizons divers, qui possèdent un DUT d'Informatique ou de Génie électrique et qui ont tous suivi un enseignement d'anglais de spécialité ; mais ce ne sont pas encore des experts dans leur domaine d'intérêt.

4 Il est nécessaire de connaître les besoins et les statuts futurs des étudiants, car ces critères détermineront le contenu du cours et l'approche didactique. On peut aisément supposer qu'ils seront amenés à se documenter sur du matériel technique, à puiser leur savoir dans des ouvrages rédigés en langue anglaise. Il convient donc de privilégier la compréhension écrite, étant donné l'horaire qui leur est imparti : 48 heures/année soit 2 heures par semaine. 
Leur programme est dense et comporte plusieurs sujets d'étude ; j'ai choisi de privilégier l'image par goût personnel. D'une part, j'ai travaillé en collaboration avec un informaticien (Michel Eboueya en l'occurrence) à la rédaction d'un rapport technique présentant les termes et concepts en visualisation scientifique; d'autre part, l'infographie se situe au carrefour de l'art et de la science, alliant la perfection de l'image et la haute technicité.

6 Mais point de programme d'anglais scientifique ni de manuel! Tout est à faire. Le terrain n'est pas encore défriché, nous constituerons notre corpus.

7 La langue de spécialité sert à la documentation et à la communication, mais elle comporte ses propres registres linguistiques et elle est en perpétuelle évolution. Les finalités didactiques sont là, qui demandent que l'étudiant puisse lire et comprendre le discours spécialisé et qu'il puisse ensuite s'approprier la langue de spécialité. Son acquisition est un objectif honorable, mais reste un processus difficile.

8 Alors, comment procéder? Dans le cas présent, l'enseignement de l'anglais s'inscrit dans les limites d'un domaine étroit et pointu: il leur faudra maîtriser un nouveau registre terminologique. L'élaboration d'un glossaire spécialisé par le groupe classe s'intégrant dans l'étude de textes les plus divers peut constituer un point de départ: les étudiants seront amenés à restituer, organiser et transmettre des données de haute spécificité, mais aussi analyseront les informations que peut apporter un texte.

9 Avant d'envisager les activités possibles pour mener à bien cette tâche et les acquisitions que l'on peut espérer, je voudrais centrer ma réflexion sur l'intérêt pédagogique d'une telle démarche.

\section{Intérêt pédagogique}

10 Un travail préalable consiste à impliquer les étudiants dans ce projet que nous mènerons conjointement; ils vont apporter dans un domaine de pointe une contribution qui fait défaut pour l'instant. Si une langue de spécialité n'est évidemment pas que de la terminologie, c'est aussi de la terminologie : elle ne doit donc pas être sous-estimée.

11 La terminologie peut être considérée comme un outil linguistique utile dans la rédaction ou la traduction de documents scientifiques. Elle répond au besoin de normes et à celui de diffusion : en effet, c'est l'un des vecteurs principaux de transmission de l'information.

En l'occurrence, elle sera un précieux outil didactique. Nous pouvons nous interroger sur la manière d'aborder ce vocabulaire spécifique. Il est possible de le faire par le biais d'une liste de termes avec leur traduction et éventuellement une définition. Mais alors, comment réactiver le vocabulaire lorsqu'il est détaché du texte?

13 La terminologie est bien fixée au contexte, par conséquent il est indispensable de la présenter dans différents textes et genres, de l'étudier dans son tissu vivant et de tenir compte de la définition. Nous aurons tôt fait de découvrir la belle diversité des usages pour un terme donné. Prenons le terme "frame»: en langue générale, il recouvre plusieurs signifiés; en anglais informatique, selon le domaine, il peut vouloir dire: " cadre », ou " trame » en infographie, ou « schéma » en intelligence artificielle. Ainsi, si les étudiants travaillent sur des termes et leur définition, et là je reprendrai une citation de J.-M. Baïssus, «à partir de leurs propres expériences d'extraction du sens dans des contextes signifiants ", ils vont sans aucun doute les mémoriser plus facilement. 
14 Les faire travailler sur la terminologie, c'est aussi les inviter à s'interroger sur les termes et expressions qui deviennent objets d'étude; c'est leur demander d'étudier des faits linguistiques et de les mettre en position de chercheur. C'est leur permettre de découvrir les constantes du discours de spécialité et d'aborder les processus rhétoriques remarquablement développés dans le livre de Louis Trimble (1985). Et c'est enfin les aider à améliorer leur qualité de lecture.

Quel sera le support?

16 Je vous propose un carré magique du support de cours: la sélection de documents répondra à quatre critères essentiels (voir figure 1).

Figure 1. Carré magique du support de cours

\begin{tabular}{|l|c|}
\hline I'authenticité & l'actualité \\
\hline l'intérêt & la diversité \\
& \\
\hline
\end{tabular}

17 Il est important de leur proposer des textes authentiques tant par leur contenu scientifique que linguistique. Nous allons donc nous appuyer sur des documents extraits de livres originaux, de revues et autres articles, riches de termes spécifiques ou d'éléments lexicaux surprenants (nombreuses sont les questions que soulèvent des items tels que "whilst », «pitfall », « to encompass », ou «fraught with »).»En utilisant ces textes, les étudiants acquièrent des connaissances nouvelles en contexte réaliste, à défaut de situation réelle. Certes, pour céder à la facilité ou vaincre l'appréhension, j'aurais pu choisir des textes pour lesquels je me sentais relativement compétente, espérant ainsi apporter une appréciation pertinente et efficace, mais j'aurais encouru le risque de démotiver les étudiants qui souhaitent accroitre leurs compétences au niveau de la langue pleinement professionnelle.

18 Les critères suivants ont également leur importance dans le choix des documents :

19 - évitons les textes obsolètes, les innovations se multiplient rapidement dans ce type de discipline.

20 - néanmoins, abstenons-nous d'anticiper en introduisant des informations nouvelles (on est en mesure de reconnaître ce que l'on attend ou ce que l'on connaît déjà); il est préférable d'étudier des documents complémentaires à la formation qu'ils reçoivent, en collaboration intelligente avec nos collègues scientifiques.

21 - il faut tenir compte de la longueur du texte et de son degré de technicité.

22 - le corpus inclut des écrits divers, présentant des niveaux d'énonciation différents : ce sont des textes spécialisés (publications scientifiques, extraits de manuels didactiques, revues professionnelles d'information), mais aussi des textes de vulgarisation. Le texte spécialisé est rédigé dans une langue plus technique et plus sèche alors que le discours d'un texte de vulgarisation donnera l'occasion d'une discussion, d'un débat ou d'une rédaction de synthèse. 


\section{.} elle est l'essence et l'issue de la langue de spécialité, le cœur et le noyau (the core and the kernel); c'est le noyau qui va donner naissance à d'autres écrits et apporter la connaissance, en permettant à la science de s'exprimer, en permettant ainsi l'existence de la langue scientifique, mais c'est aussi le cœur de la langue de spécialité, vers lequel il faut tendre puisqu'elle assure le dialogue. Très schématiquement, on pourrait représenter la langue de spécialité de cette manière (voir figure 2).

Figure 2

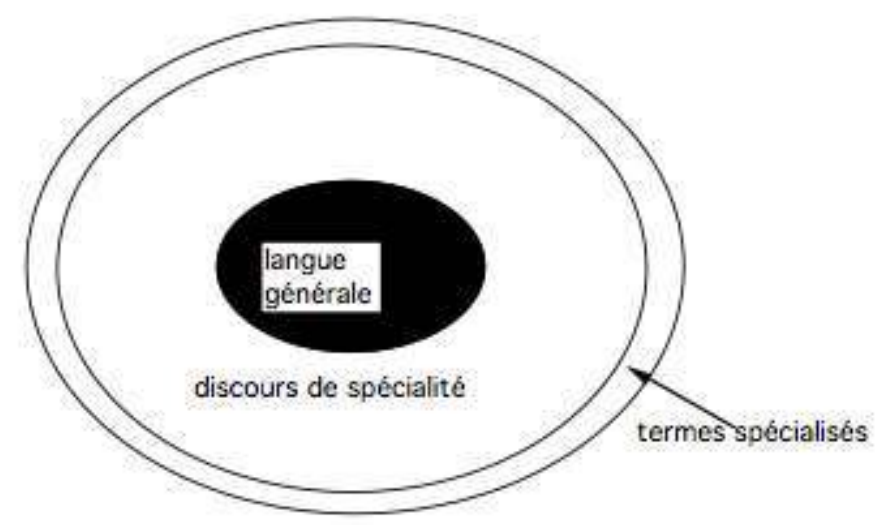

Si nous admettons cette représentation, nous devons alors adapter notre approche pour que les étudiants puissent acquérir une compétence professionnelle et finalement apprendre la langue anglaise dans son intégralité, la langue tout court. 
28 Si l'on reconnaît que notre apprenant a besoin d'assimiler les concepts et termes spécialisés, on doit surtout le former au discours scientifique qui relève de la langue générale, et le convaincre qu'il doit vivre et intégrer la langue en situation (avec enthousiasme si possible). On peut procéder par niveaux, par strates, en délimitant bien les différents exercices; c'est ce que j'appellerai la méthode du pas-à-pas. On peut préférer la méthode parallèle et choisir d'intégrer globalement la découverte de la langue de spécialité et de la langue générale au sein d'une même unité, croiser tous les types d'exercices possibles pour aboutir à une variété assez tentante pour l'étudiant le plus gourmand qui, constamment renvoyé au texte dans sa globalité, l'assimilera correctement et en gardera toute la saveur.

Nous pouvons à présent envisager les séquences pédagogiques, et aborder les exercices d'apprentissage.

\section{Activités et acquisitions}

30 L'auteur d'un document scientifique exprime des idées ou expose des théories à l'aide de concepts très précis qui permettent la communication d'informations (verbales ou écrites). Ainsi que le souligne J. Gallais-Hamonno (1979),

la connaissance de la théorie (c'est-à-dire des traits accordés par l'analyse aux termes qui désignent les concepts) permet de comprendre un texte scientifique.

Pour constituer le glossaire, les étudiants vont dans un premier temps effectuer une collecte de termes, les sélectionner, étudier le nombre d'occurrences, puis dans un deuxième temps trouver une traduction juste ou proposer une définition, expliquer et justifier. Nous orchestrons des activités de groupes, ce qui peut être plus rassurant pour l'étudiant un peu timide; et nous favorisons ainsi les discussions. Il est bon de leur proposer aussi des activités individuelles au cours desquelles ils travaillent sur des exercices de substitution, ou de mise en correspondance, ou des exercices lacunaires. Cet ensemble d'exercices ne prétend ni à l'originalité ni à l'exhaustivité.

Toutefois, ces différentes tâches contribuent à des acquisitions générales: en effet, les étudiants apprennent à définir les limites d'un travail, à trouver les ressources et les repères dans un domaine, ils développent leur faculté de raisonnement critique et prennent en charge leur propre apprentissage et surtout découvrent les potentialités qui sont en eux.

Au niveau scientifique, ils étudient leur domaine d'intérêt, et en manipulant les termes, ils les fixent.

Les acquisitions linguistiques sont importantes:

- ils apprennent à former les termes et à les définir ;

36 - à utiliser le dictionnaire unilingue de préférence (qui peut être un outil étymologique : il est intéressant de remonter aux sources) et à choisir à bon escient ;

37 - à développer des facilités de déduction : le sens des termes est induit par le texte ;

38 - à établir des relations d'analogie ou sémantiques ;

39 - à reconnaître les termes " purs et durs " propres à la discipline ou les néologismes, par exemple : "aliasing" (crénelage ou aliassage) ; « imagery» (imagerie) ; 
40 - à se référer à la langue générale : les exemples sont multiples : «to crop» (recadrer); "shading» (ombrage) ou même " heuristics» (heuristique).

41 Cependant, si le lexique spécialisé joue un rôle important, il n'est peut-être que la partie visible de l'iceberg. Les étudiants lisent les concepts, mais leurs difficultés de compréhension d'un texte scientifique sont liées à la nature du discours et de son fonctionnement.

On ne peut éviter certains aspects linguistiques spécifiques, communs au discours scientifique où les mêmes expressions et structures reviennent (connecteurs, voix passive, modaux, comparatifs, noms composés). Là encore, il est souhaitable de leur donner diverses occasions de traiter les problèmes syntaxiques en ayant recours à des exercices de substitution, de reformulation, de création (de noms composés par exemple), et même de traduction pour vérifier l'assimilation et l'expression. Le passif sera étudié dans un contexte authentique : on évitera la transformation de phrases isolées, et l'on préférera la rédaction d'un paragraphe sur un processus connu des étudiants en leur présentant éventuellement une liste de mots à utiliser. Que de contresens et d'ambiguïtés si l'apprenant ne parvient pas à repérer un nom composé ; je vous propose la phrase suivante : " the incident ray, the reflected ray and the surface normal at the point of incidence all lie in the same plane » ("surface normal » signifie « la perpendiculaire à la surface »).

Il sera possible d'effectuer des tâches qui tendent à faciliter l'appropriation linguistique tout en traitant tel ou tel aspect du discours: le travail sur les connecteurs les sensibilisera à l'organisation logique d'un texte, aux différentes articulations, à l'argumentation et leur permettra d'analyser les processus rhétoriques et les techniques correspondantes, en résumé la macro-structure du texte.

Pour vérifier la qualité de leur compréhension, on leur demande de différencier les types de texte, de prendre des notes dont ils disposent pour compléter un paragraphe, pour contracter le texte ou rédiger un résumé. Des exercices tels que la recherche de l'information essentielle ou d'éléments ponctuels sont naturellement désignés pour leur permettre de maitriser la lecture et au-delà de développer leurs compétences de lecture extensive : suscitons leur intérêt et incitons-les à consulter d'autres documents en leur faisant découvrir qu'un texte peut être abordable, quelle qu'en soit la longueur ou la teneur du contenu. Je ne passerai pas en revue toutes les activités possibles que chacun d'entre nous a pu pratiquer.

\section{Conclusion}

45 L'idéal est sans doute de les encourager à une autonomie et une efficacité sur le plan du processus d'acquisition des connaissances, mais il n'en reste pas moins vrai qu'entre le professeur et les étudiants doit s'établir une sorte de synergie, voire de complicité.

Il est un peu prématuré de dresser un bilan après cette première année de mise en œuvre ; c'est un travail qui n'en est qu'à ses balbutiements. Par ailleurs, j'espère n'être arrivée à aucune conclusion hâtive, simplificatrice ou réductrice; j'ai eu simplement envie de livrer dans ce propos des réflexions sur une démarche exploratoire.

Il reste que cette expérience a motivé les étudiants, qu'ils se sont investis et il reste enfin un grand enthousiasme de leur part et de la part de l'auteur de ces lignes. C'est sur cette note positive que je veux terminer. 


\section{BIBLIOGRAPHIE}

Baïssus, Jean-Marie. 1984. « Pour une pédagogie du sens ». Cahiers de l'ILSER 3, 27-36.

Dudley-Evans, T. 1992. «L'enseignement en équipe et le degré de spécialisation de l'enseignement en ESP ». Actes du XIII Colloque du GERAS. ASp 1, 1-9.

Eboueya, M. et N. Pasternak Eboueya. 1992. «Quelques-uns des termes et concepts utilisés en visualisation scientifique ». Rapport interne du CRISTEL janvier.

Gallais-Hamonno, J. 1979. The Language of Basic Economics: manuel d'anglais économique. Paris : Dunod.

Trimble, L. 1985. English for Science and Technology: A discourse approach. Cambridge : Cambridge University Press.

\section{AUTEUR}

\section{NADINE PASTERNAK-EBOUEYA}

Université de Poitiers, site de La Rochelle, IUT Informatique.neboueya@univ-lr.fr 